\title{
A low complexity peak-to-average power ratio reduction scheme based on selected mapping and radix-II IFFT in OFDM systems
}

\author{
Mohsen Kazemian $^{1}$ - Pooria Varahram ${ }^{2}$ - S. J. Hashim ${ }^{1}$. \\ Borhanuddin M. Ali ${ }^{1,3}$. Ronan Farrell ${ }^{2}$
}

Published online: 3 May 2017

(C) Springer Science+Business Media New York 2017

\begin{abstract}
Because of its lack of feedback process and the simplicity of its searching algorithm, conventional selected mapping (CSLM) is an efficient peak-to-average power ratio (PAPR) reduction technique in orthogonal frequency division multiplexing systems compared to the present techniques such as partial transmit sequence and active constellation extension. The requirement for large numbers of inverse fast Fourier transform (IFFT) blocks to provide desired PAPR reduction performance is introduced as the most significant drawback of the CSLM. This paper uses the special structure of an N-point radix-II IFFT in the CSLM and proposes a low complexity method to reduce the redundant calculations with almost the same PAPR reduction, bit-error rate, and power spectral density performances as those of the CSLM. The simulation results show that the computational complexity
\end{abstract}

Mohsen Kazemian

mrmohsenkazemian@gmail.com

Pooria Varahram

pooria.varahram@nuim.ie

S. J. Hashim

sjh@upm.edu.my

Borhanuddin M. Ali

borhan@upm.edu.my

Ronan Farrell

ronan.farrell@nuim.ie

1 Department of Computer and Communications Systems Engineering, Universiti Putra Malaysia, Serdang, Selangor, Malaysia

2 Department of Electronic Engineering, Callan Institute, National University of Ireland, Kildare, Ireland

3 Wireless \& Photonics Research Centre, Faculty of Engineering, Universiti Putra Malaysia, Serdang, Selangor, Malaysia is reduced by at least $46.8 \%$ compared to that of the CSLM with approximately the same PAPR performance.

\section{Keywords OFDM · IFFT · PAPR · RADIX-II · CSLM}

\section{Introduction}

A new generation of wireless communication systems should be able to meet various important specifications such as the ability to transmit at a high data rate with emphatic constraints on power consumption, and bandwidth seizure. Hence, it is necessary to adopt power-efficient and M-ary modulation schemes with a high spectral efficiency, including quadrature amplitude modulation (QAM) in conjunction with orthogonal frequency division multiplexing (OFDM). Because of the benefits of OFDM, the use of this technique for cellular mobile radio standards, long-term evolution (LTE), and future wireless standards is prevalent. When considering several research reports, a major drawback of OFDM signals is a high peak-to-average power ratio (PAPR) because a large PAPR leads to in-band distortion, out-of-band radiation, efficiency degradation, and low battery life. Therefore, it usually affects mobile consumer electronics, such as smart phones, laptops, and tablet PCs in uplink communications. An enormous amount of research has considered the problem of the PAPR inherent in OFDM signals. Various techniques have been proposed based on different metrics such as the mean squared error (MSE), inter modulation distortion (IMD) [1], complementary cumulative distribution function (CCDF), peak interference-to-carrier ratio (PICR), and bit-error rate (BER). Partial transmit sequence (PTS) $[2,3]$ is a distortionless phase optimization technique performed using the optimal combination of signal subblocks. However, PTS suffers the drawback of the high computational complexity based on the sub block numbers and the feedback procedure. 
Interleaving [4], active constellation extension (ACE), and conventional selected mapping (CSLM) [5] are other popular techniques. Due to its lack of feedback process, CSLM is considered to be one of the most efficient distortion-less PAPR reduction schemes [2]. CSLM is based on the multiplication of random phase sequences by the input signal, in the frequency domain, and the selection of the signal with the smallest PAPR. The requirement for large numbers of inverse fast Fourier transform (IFFT) blocks for finding the optimum phase sequence is the most important challenge addressed by recent research efforts. A few researchers have attempted to address this particular drawback by minimizing the number of IFFT blocks [6].

Li's method [5] includes certain base vectors, and their cyclically shifted versions. Despite the high complexity reduction achievement, its PAPR reduction is inferior to that of CSLM. Kim [7] proposed a new CSLM technique using a cyclic-shifted IFFT. Although this technique reduces the complexity in comparison to the CSLM, a high number of subblocks are nevertheless needed to provide sufficient PAPR reduction. Due to the generation of a high number of candidate signals, high processing time is a drawback of the two above-mentioned methods.

Newer transmission formats, such as code-division multiple access (CDMA), and OFDM systems are especially vulnerable to power amplifier (PA) nonlinearities due to their high PAPR, corresponding to large fluctuations in their signal envelopes. To comply with spectral masks imposed by regulatory bodies and to reduce BER, PA linearization must be applied. Predistortion techniques have been proposed as a potential solution to address the nonlinear effects. These techniques attempt to introduce inverse nonlinearities that can compensate for the distortions generated by the nonlinear amplifier. The predistortion must be adaptive because of variations in power amplifier nonlinearity with time, temperature, different operating channels, and so on. Figure 1 shows the OFDM transmitter structure in terms of PAPR reduction, digital predistortion (DPD), and PA blocks.

In this paper, a novel low complexity SLM scheme using a single IFFT operation is proposed. The proposed approach is based on detecting the redundant calculations of the IFFT block, and saving those for the subsequent stages; hence, the need to perform repetitive calculations is eliminated. This method excels in PAPR reduction with nearly the same complexity; in addition, it excels in terms of complexity reduction with nearly the same PAPR reduction compared to the CSLM. The simulation results show that the proposed technique does not adversely affect the BER performance or the power spectral density (PSD) of the PA; thus, the BER performance and the PSD will be the same as those of the CSLM. However, the great achievement of this approach is computational complexity reduction.



Fig. 1 Block diagram of an OFDM transmitter

This paper is organized as follows: Sect. 2 includes the definitions of CSLM, PAPR and the IFFT operation. The proposed scheme, low complexity radix-2 SLM (LR-SLM), is described in Sect. 3. A comparison of the proposed method with other methods in terms of computational complexity is discussed in Sect. 4. Simulation results of the proposed method are discussed in Sect. 5 and followed by the conclusion in Sect. 6. Additionally, the appendix presents two examples of the proposed scheme.

\section{Definitions}

\subsection{PAPR and CSLM}

In this paper, $X=\{X(0), X(1), \ldots, X(N-1)\}$ and $x=$ $\{x(0), x(1), \ldots, x(N-1)\}$ are considered as the signals in the frequency domain and time domain, respectively. The relation between the input symbol sequence $X$, which is usually modulated using phase-shift keying (PSK) or QAM, and the OFDM signal sequence $x$ is expressed by IFFT as

$x(n)=\frac{1}{\sqrt{N}} \sum_{p=0}^{N-1} X(p) W^{-n p}$

where $W=e^{-j((2 \pi) / N)}, n \in\{0,1, \ldots, N-1\}$, and $N$ is the number of subcarriers.

The PAPR is a measure that is generally used to quantify the envelope variations in the multicarrier signals, using a minimum of four times oversampling [2] and can be defined as

$P A P R=\frac{\max _{0 \leq n \leq N-1}\left[|x(n)|^{2}\right]}{E\left[|x(n)|^{2}\right]}$

where $E[\cdot]$ denotes the expectation value.

Figure 2 shows the CSLM scheme, which uses the original input signal sequence, $V$ times. The CSLM generates $V$ distinct phase rotation vectors $b^{v}$, which are known to the transmitter and receiver, where $b^{v}=\left\{b^{v}(0), b^{v}(1), \ldots, b^{v}(N-\right.$ $1)\}, b^{v}(m) \in\{1,-1, j,-j\}$, and $0 \leq v \leq V-1$. 


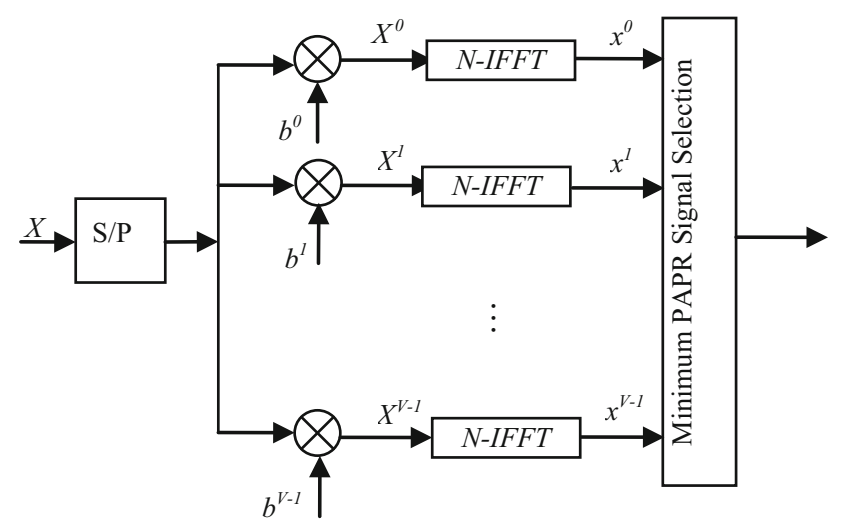

Fig. 2 Block diagram of conventional SLM (CSLM) scheme

The input symbol sequence $X$ is multiplied element-wise by the phase vector, as $X^{v}(\mathrm{~m})=X(\mathrm{~m}) \cdot \mathrm{b}^{v}(m)$. After passing the signal through the IFFT blocks, $x$ as the time-domain signal is retrieved, where $x^{v}=\operatorname{IFFT}\left(X^{v}\right)$. Finally, the PAPR values of all $V$ signals are calculated, and the signal with the minimum PAPR is selected as follows:

$\bar{v}=\underset{v}{\arg \min }\left(\frac{\max \left|x^{v}(n)\right|^{2}}{E\left[\left|x^{v}(n)\right|^{2}\right]}\right)$

where $E[\cdot]$ represents the expected value operator and $b^{\bar{v}}$ is the optimum phase sequence, which is specified as the side information and is transmitted to the receiver [3].

\subsection{Inverse fast Fourier transform}

The basic IFFT equations are used in the proposed approach; hence, they will be described in this section. The basic IDFT equation is expressed by

$x(d)=\frac{1}{\sqrt{N}} \sum_{p=0}^{N-1} X(p) e^{j\left(\frac{2 \pi}{N}\right)} d p$

after splitting into even and odd parts,

$$
\begin{aligned}
x(d) & =\sum_{p=0}^{\frac{N}{2}-1} X_{2 p} e^{j \frac{2 \pi}{N}(2 p) d}+\sum_{p=0}^{\frac{N}{2}-1} X_{2 p+1} e^{j \frac{2 \pi}{N}(2 p+1) d} \\
& =\sum_{p=0}^{\frac{N}{2}-1} X_{2 p} e^{j \frac{2 \pi}{N / 2} p d}+e^{j \frac{2 \pi}{N} d} \sum_{p=0}^{\frac{N}{2}-1} X_{2 p+1} e^{j \frac{2 \pi}{N / 2} p d} \\
& =G_{d}+W^{d} H_{d} \\
x(d & +N / 2)=\sum_{p=0}^{\frac{N}{2}-1} X_{2 p} e^{j \frac{2 \pi}{N / 2} p d}-e^{j \frac{2 \pi}{N} p} \sum_{p=0}^{\frac{N}{2}-1} X_{2 p+1} e^{j \frac{2 \pi}{N / 2} p d} \\
& =G_{d}-W^{d} H_{d}
\end{aligned}
$$

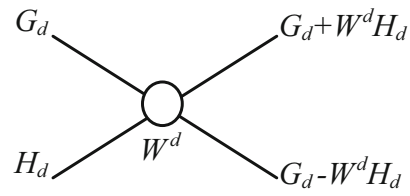

Fig. 3 Radix-2 IFFT butterfly structure

where

$G_{d}=\sum_{p=0}^{\frac{N}{2}-1} X_{2 p} e^{j \frac{2 \pi}{N / 2} p d}, H_{d}=\sum_{p=0}^{\frac{N}{2}-1} X_{2 p+1} e^{j \frac{2 \pi}{N / 2} p d}$

$W^{d}=e^{j \frac{2 \pi}{N} d}$ and $d \in\{0,1, \ldots, N / 2-1\}$. Figure 3 shows the above equations defined in the radix -2 butterfly structure.

\section{Proposed method}

This section details the LR-SLM scheme and relevant equations. As previously highlighted, for CSLM and SLM-based methods, the input signal samples are multiplied elementwise by random phase sequences. The simulation proves that there is no need to create $N$ random phases for each IFFT block as in CSLM when the same result can be achieved using N/4 different phases for each block if the phases are different from each other. Intuitively, in CSLM and SLMbased methods, the phase sequences are randomly selected and should only be selected from $(\mathrm{j},-\mathrm{j}, 1,-1)$; therefore, there are 4 ! (i.e., 24) different possible conditions. Previous works did not consider similar random phase sequences for each IFFT blocks, whereas the proposed method looks for similar calculations based on the similar phase sequences, saves, and uses them for the following search procedures. This approach ensures different phase sequence generation, which eliminates repetitive inner IFFT calculations to reduce the computational complexity, and processing time in addition to improving the PAPR reduction performance, and power efficiency, which decreases the cost of the consumer electronics.

As shown in Fig. 4, each $N$-point radix-2 IFFT can be partitioned into 4 groups of data samples such that 2 of these groups carry even-numbered data samples, and the other 2 groups carry odd-numbered samples. $X_{m_{1}}$ and $X_{m_{3}}$ are the odd samples, and $X_{m_{2}}$ and $X_{m_{4}}$ are the even samples as follows:

$$
\begin{aligned}
& m_{1}=4\left(N / 4-m^{\prime}\right)-1 \\
& m_{2}=4\left(N / 4-m^{\prime}\right)-2 \\
& m_{3}=4\left(N / 4-m^{\prime}\right)-3 \\
& m_{4}=4\left(N / 4-m^{\prime}\right)-4
\end{aligned}
$$




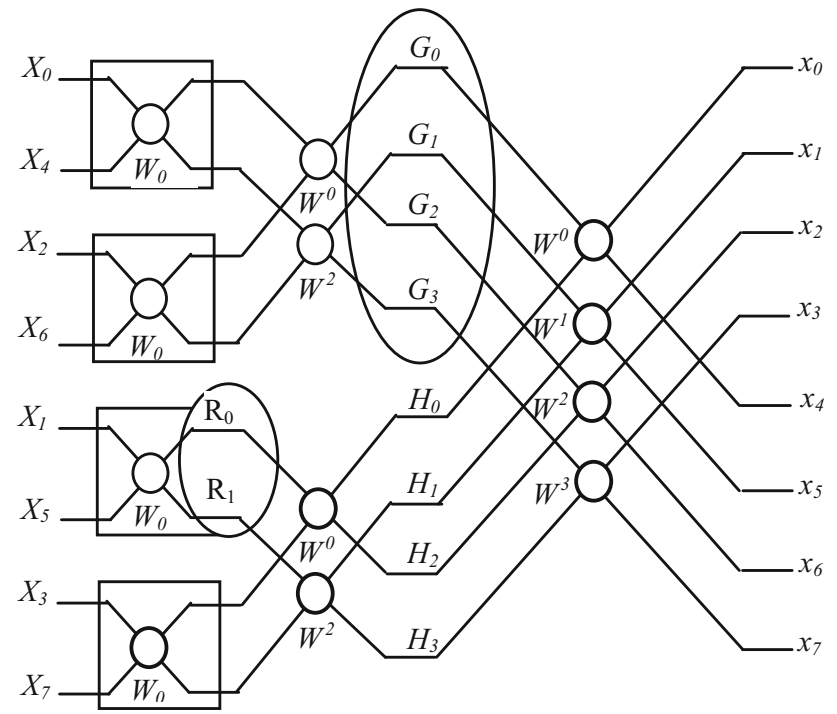

Fig. 4 Illustration of the proposed scheme based on saved parameters for an 8 point radix-2 IFFT

where $m^{\prime}=0,1, \ldots, \frac{N}{4}-1$. The new matrix $X^{\prime}$ is defined by

$X^{\prime}=\left[\begin{array}{llll}X_{m_{1}} & X_{m_{2}} & X_{m_{3}} & X_{m_{4}} \\ X_{m_{1}} & X_{m_{2}} & X_{m_{3}} & X_{m_{4}} \\ \vdots & \vdots & \vdots & \vdots \\ X_{m_{1}} & X_{m_{2}} & X_{m_{3}} & X_{m_{4}}\end{array}\right]_{K \times N} 4 \leq K \leq 16$

where $K$ is the number of random phase sequences. It should be highlighted that in the CSLM method, the number of random phase sequences is the same as the number of IFFT blocks (i.e., $K=V$ ), whereas in the proposed method, the number of IFFT blocks is reduced to one (i.e., $V=1$ and $K$ is the number of phase sequences). The proposed phase sequence matrix $b_{i}$ is a circular right shift (CRS) of the matrix $B$ by $(i-1) \frac{N}{4}$ positions and can be extended to $B^{\prime}$ as expressed by

$$
B^{\prime}=\left[\begin{array}{l}
b_{1} \\
b_{3} \\
b_{2} \\
b_{4}
\end{array}\right]_{K \times N}, b_{i}=\{C R S(B)\}_{(i-1) \frac{N}{4}}, i \in\{1,2,3,4\}
$$

where $B=\left[\begin{array}{lr}\overbrace{1,1, \ldots, 1}^{N / 4} & 1,1, \ldots 1 \\ -1,-1, \ldots,-1 & 1,1, \ldots 1 \\ j, j, \ldots, j & 1,1, \ldots 1 \\ -j,-j, \ldots, j & 1,1, \ldots 1\end{array}\right]_{4 \times N}$. For example, with $K=4, B^{\prime}=b_{1}$. Additionally, 2 matrices $c_{1}$ and $c_{2}$ can be generated using the $b_{i}$ matrices as $c_{1}=b_{1} \circ b_{3}$

$c_{2}=b_{2} \circ b_{4}$

where the sign $\circ$ indicates element-wise multiplication. Due to the multiplication of $K=V$ random phase sequences with the input signal, $K$ IFFT blocks are needed in the CSLM, whereas only one IFFT block is needed in the proposed method, using $K$ phase sequences. Because of the novel structure of the $X^{\prime}$, and $B^{\prime}$ matrices, only $N / 4$ and $N / 2$ phases have to be changed when using the $b_{i}$ and $c$ matrices, respectively. Therefore, the novel approach is based on saving calculations related to the fixed phases and applying them for the following search procedures, to reduce the repetitive calculations. The input of the IFFT block for the above-mentioned matrices is given by

$$
\begin{aligned}
& Y_{1}=X^{\prime} \circ b_{i} \quad(K=4) \\
& Y_{2}=X^{\prime} \circ c_{i^{\prime}} \quad i^{\prime} \in\{1,2\} \\
& Y_{3}=X^{\prime} \circ B^{\prime} \quad 4 \leq K \leq 16
\end{aligned}
$$

Note that the matrix $B^{\prime}$ has $K$ rows selected by the user. Using $K=4$, the matrix includes $b_{1}$, using $K=8$, it includes $b_{1}$, and $b_{3}$, and the same explanation for $K=12$ and $K=16$. Hence, the matrices $Y_{1}$ and $Y_{2}$ have 4 rows when the number of rows for matrix $Y_{3}$ is $K$.

The first row is the first input for the IFFT, and subsequently, the PAPR value is obtained at the IFFT output. The second row of the matrix is the next IFFT input, whereas the internal multiplication and addition calculations of the IFFT block are the same as for the previous row, except for the $N / 4$ input samples by $b_{i}$ and $c_{i \prime}$, and $N / 2$ input samples for $B^{\prime}$. The same procedure applies to the other rows. The operation of each row of the proposed matrices on the input signal through the one IFFT is the same as one IFFT block using random phase sequences in the CSLM method. If $y_{j}^{u}=\operatorname{IFFT}\left(Y_{j}^{u}\right)$ and $y_{j}^{u}=\left\{y_{j}^{u}(0), y_{j}^{u}(1), \ldots, y_{j}^{u}(N-1)\right\}$, where $Y_{j}^{u}$ is the $u_{t h}$ row of the matrix $Y_{j}$ and $j \in\{1,2,3\}$, then the signal with the minimum PAPR is selected as follows:

$\bar{u}=\underset{u}{\arg \min }\left(\frac{\max _{0 \leq r \leq N-1}\left|y_{j}^{u}(r)\right|^{2}}{E\left[\left|y_{j}^{u}(r)\right|^{2}\right]}\right), \quad 1 \leq u \leq K$

where $j$ is dependent on the selected matrix and $\bar{u}$ is the optimum phase sequence. As shown in Fig. 4, by selecting $b_{1}, b_{3}$ or $c_{1}$ as the phase matrix, $G_{d}$, and by selecting $b_{2}, b_{4}$ or $c_{2}$ as the phase matrix, $H_{d}$ becomes fixed. Therefore, after the internal IFFT calculations for the first row, $G_{d}$ and $H_{d}$ can be saved and used for all other rows. In addition, using the $b_{i}$ matrix, the other parameters as $R_{l+N / 8}$ and $R_{l}$, can be 
Table 1 Definition of the saved parameters in the first row, and used in the subsequent rows

\begin{tabular}{ll}
\hline Selected phase matrix & Saved parameters \\
\hline$b_{1}$ & $G_{d}, R_{l+N / 8}, R_{l}(q=3)$ \\
$b_{2}$ & $H_{d}, R_{l+N / 8}, R_{l}(q=2)$ \\
$b_{3}$ & $G_{d}, R_{l+N / 8}, R_{l}(q=1)$ \\
$b_{4}$ & $H_{d}, R_{l+N / 8}, R_{l}(q=4)$ \\
$c_{1}$ & $G_{d}$ \\
$c_{2}$ & $H_{d}$ \\
\hline
\end{tabular}

saved, which are defined as

$R_{l}=\alpha_{l}+W^{4 l} \beta_{l}$

$R_{l+N / 8}=\alpha_{l}-W^{4 l} \beta_{l}$

$\alpha_{l}=\sum_{\Gamma=0}^{\frac{N}{8}-1} X_{\Re} e^{j \frac{2 \pi}{N / 8} \Gamma l}$

$\beta_{l}=\sum_{\Gamma=0}^{\frac{N}{8}-1} X_{\mathfrak{R}^{\prime}} e^{j \frac{2 \pi}{N / 8} \Gamma l}$

where $\Re=4\left(\frac{N}{4}-2 n^{\prime}\right)-q, \Re^{\prime}=4\left(\frac{N}{4}-2 n^{\prime}-1\right)-q$, $0 \leq n^{\prime} \leq \frac{N}{8}-1, q \in\{1,2,3,4\}, 0 \leq l \leq \frac{N}{8}-1$ and, $W^{4 l}=$ $e^{j \frac{8 \pi}{N} l}$. Table 1 shows the parameters that are calculated once, saved, and subsequently used for the other rows. Referring to (12), $b_{1}$ affects only the last group of the input samples of an IFFT block; hence, as shown in Fig. $4, G_{d}, R_{0}$ and $R_{1}$ are the parameters that should be saved using $b_{1}$ as the phase matrix of an 8-point IFFT block. Figure 5 illustrates the proposed block diagram used to find the optimum phase sequence of an 8-point IFFT, using $b_{1}$.

The input samples are definitely changed due to the special phases of $b_{1}$. Thus, $N / 4$ changes areensured. In addition, the parameters $G_{d}, R_{0}$ and $R_{1}$ are fixed and can be used for the following rows. In contrast to the CSLM, all of the internal IFFT calculations should be performed for each random phase sequence. Additionally, the side information transmission drawback is further solved by the proposed approach. Referring to (18), the side information is the row number of the proposed matrix, which includes the optimum phase sequence. Therefore, the large matrix as the CSLM side information is replaced by simply the number of the particular row of the matrix $B^{\prime}$ which includes the optimum phase sequences.

\section{Computational complexity}

The computational complexity reduction in the proposed scheme is based on saving some inner calculations of the

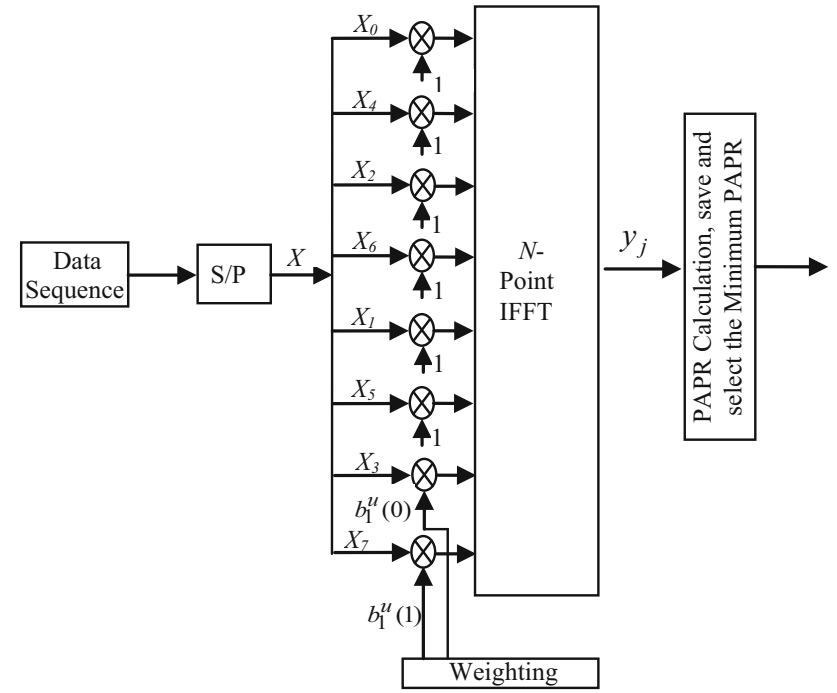

Fig. 5 The proposed method for an 8-point IFFT, using $b_{1}$, where $b_{1}^{u} \in\{1,-1, j,-j\}[8]$

IFFT, which leads to the elimination of redundant multiplication and addition operations. Therefore, to compare the proposed scheme with the CSLM, the number of saved multiplication and addition operations for each IFFT block is analyzed.

The numbers of multiplication and addition operations for each general IFFT block are defined as

$\begin{aligned} I_{I F F T_{M U L}} & =\frac{N}{2} \log _{2} N \\ \text { IFFT }_{\text {Add }} & =N \log _{2} N\end{aligned}$

If $N / 4$ input samples in the proposed method are changed using the $b_{i}$ matrices, the numbers of multiplications and additions for each IFFT block are reduced and described by:

$$
\begin{aligned}
& X_{I F F T_{M U L}}=\frac{N}{8} \log _{2} N+\frac{N}{2} \\
& X_{I F F T_{\text {Add }}}=\frac{N}{4} \log _{2} N+N
\end{aligned}
$$

Additionally, $Y_{I F F T_{M U L}}$ and $Y_{I F F T_{\text {Add }}}$ are the numbers of multiplication and addition operations of each IFFT block, respectively, using the $c_{i}$ matrices, and are defined as

$$
\begin{aligned}
Y_{I F F T_{M U L}} & =\frac{N}{4} \log _{2} N+\frac{N}{4} \\
Y_{I F F T_{\text {Add }}} & =\frac{N}{2} \log _{2} N+\frac{N}{2}
\end{aligned}
$$

Table 2 gives the required numbers of multiplications and additions for each IFFT block used in the CSLM and also in the proposed method, using the $b_{i}$ and $c_{i}$ matrices. For example, the number of multiplications of each IFFT block 
Table 2 A comparison on the numbers of multiplication and addition operations for one IFFT between CSLM and LR-SLM

\begin{tabular}{lccccc}
\hline & $\mathrm{N}=8$ & $\mathrm{~N}=16$ & $\mathrm{~N}=32$ & $\mathrm{~N}=64$ & $\mathrm{~N}=256$ \\
\hline MUL. & 12 & 32 & 80 & 192 & 1024 \\
$\quad$ FFT $_{\text {mul. }}$ & 7 & 16 & 36 & 80 & 384 \\
FFT $_{\text {mul. }}-\mathrm{b}_{\mathrm{i}}$ & 8 & 20 & 48 & 112 & 576 \\
$\quad F F T_{\text {mul. }}-c_{i}$ & 8 & & & & \\
ADD. & & & & & \\
$\quad F F T_{\text {add. }}$ & 24 & 64 & 160 & 384 & 2048 \\
$F F T_{\text {add. }}-b_{i}$ & 14 & 32 & 72 & 160 & 768 \\
$F F T_{\text {add. }}-c_{i}$ & 16 & 40 & 96 & 224 & 1152 \\
\hline
\end{tabular}

for the CSLM method when $N=32$ is the same as the proposed method for $N=64$ using the $b_{i}$ matrix.

The required multiplication and addition operations for a total of $K$ different phase sequences in the CSLM are given as

$$
\begin{aligned}
T_{K, \text { CSLM-Mul }} & =K\left(\frac{N}{2} \log _{2} N\right) \\
T_{K, \text { CSLM-Add }} & =K\left(N \log _{2} N\right)
\end{aligned}
$$

where $T_{K, C S L M-M u l}$ and $T_{K, C S L M-A d d}$ are the required numbers of multiplications and additions for $K=V$ IFFT blocks, whereas the respective numbers for the proposed scheme are given by

$$
\begin{aligned}
T_{K, \operatorname{Pr} o-M u l}= & \frac{N}{2} \log _{2} N \\
& +(K-1)\left(\frac{N}{8} \log _{2} N+\frac{N}{2}\right), 4 \leq K \leq 16 \\
T_{K, \operatorname{Pr} o-A d d}= & N \log _{2} N \\
& +(K-1)\left(\frac{N}{4} \log _{2} N+N\right), 4 \leq K \leq 16
\end{aligned}
$$

An example for $N=8$ and $K=4$ is explained in the appendix.

Table 3 compares the computational complexity of various schemes, where each IFFT operation involves $\frac{\mathrm{N}}{2} \log _{2} \mathrm{~N}$
Table 4 A comparison on the numbers of multiplications between CSLM and the proposed method for $\mathbf{a} N=256$ and $\mathbf{b} N=1024$ by different $K$ values [8]

\begin{tabular}{lrr}
\hline No. of phase seq. & CSLM & LR-SLM \\
\hline a & & \\
$K=4$ & 4096 & 2176 \\
$K=6$ & 6144 & 2944 \\
$K=8$ & 8192 & 3712 \\
$K=16$ & 16,384 & 6784 \\
$\mathrm{~b}$ & & \\
$K=4$ & 20,480 & 10,496 \\
$K=6$ & 30,720 & 14,080 \\
$K=8$ & 40,960 & 17,664 \\
$K=16$ & 81,920 & 32,000 \\
\hline
\end{tabular}

complex multiplications and $N \log _{2} N$ complex additions, where $N=2^{n^{\prime}}$, and $i^{\prime \prime}$ is the number of stages [7].

The computational complexity reduction ratio (CCRR) of the proposed technique over the CSLM is defined as [8]

$C C R R=\left(1-\frac{\text { Complexity of the LR-SLM }}{\text { Complexity of the CSLM }}\right) \times 100 \%$

Table 4 present the number of multiplications in the CSLM and the LR-SLM method for $N=256$ and $N=1024$ by $K$ different phase sequences, respectively. For $K=6$, the complexity of the CSLM is approximately the same as that of the proposed method for $K=16$ (the higher values for $K$, are not practical in CSLM scheme, due to the high complexity). Additionally, Table 5 compares the CCRR of the proposed method, CSLM, and the methods in [5] and [7] when $i^{\prime \prime}=4$. The comparison is performed with $i^{\prime \prime}=4$ to provide the nearly sufficient PAPR reduction value in most wireless systems for a proper comparison. The proposed method outperforms the CSLM and the Kim's method in terms of numbers of multiplications and additions, and the Li's method in terms of additions. The minimum complexity reduction between the CSLM and the proposed scheme is found to be $46.8 \%$.

Although the method in [5] is superior in terms of the reduction in the number of multiplications compared to
Table 3 Computational complexity of various schemes

\begin{tabular}{lll}
\hline Methods & Number of multiplications & Number of additions \\
\hline CSLM & $K \frac{N}{2} \log _{2}^{N}$ & $K N \log _{2}^{N}$ \\
Li's method [5] & $\frac{N}{2} \log _{2}^{N}$ & $N\left(\log _{2}^{N}+3 K\right)$ \\
Kim's method [7] & $\frac{N}{2} \log _{2}^{N}\left(\frac{n^{\prime}-i^{\prime \prime}(K-1)}{n^{\prime}}\right)$ & $N \log _{2}^{N}\left(\frac{n^{\prime}-i^{\prime \prime}(K-1)}{n^{\prime}}\right)$ \\
LR-SLM & $\left\{\begin{array}{c}\frac{N}{2} \log _{2} N \\
+(K-1)\left(\frac{N}{8} \log _{2} N+\frac{N}{2}\right)\end{array}\right\}$ & $\left\{\begin{array}{l}N \log _{2} N \\
+(K-1)\left(\frac{N}{4} \log _{2} N+N\right)\end{array}\right\}$ \\
\hline
\end{tabular}


Table 5 A comparison on CCRR\% between different methods for $N=256$

\begin{tabular}{|c|c|c|c|c|c|c|}
\hline & \multicolumn{2}{|c|}{ Method in [5] CCRR\% } & \multicolumn{2}{|c|}{ Method in [7] CCRR\% } & \multicolumn{2}{|c|}{ LR-SLM CCRR\% } \\
\hline & Mul. & Add. & Mul. & Add. & Mul. & Add. \\
\hline$K=4$ & 75.0 & 37.5 & 37.5 & 37.5 & 46.8 & 46.8 \\
\hline$K=6$ & 83.3 & 45.8 & 42.7 & 42.7 & 52.08 & 52.08 \\
\hline$K=8$ & 87.50 & 50.0 & 43.8 & 43.8 & 54.68 & 54.68 \\
\hline$K=16$ & 93.75 & 56.25 & 46.9 & 46.9 & 58.59 & 58.59 \\
\hline
\end{tabular}

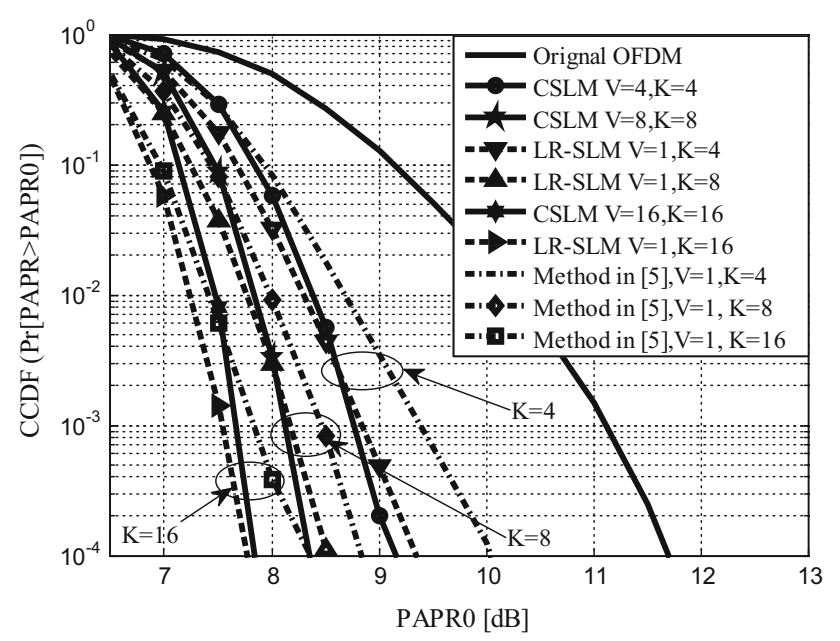

Fig. 6 A comparison between CSLM, LR-SLM, and Li's method [5] by the same $K$ values when $N=256$

CSLM and LR-SLM, its PAPR reduction performance is inferior, which is a priority in this research area (see Fig. 6).

Therefore, this method uses only one IFFT block for $K$ times (see Fig. 5). Since most of the calculations in the $K$ different times are the same, the similar calculations are saved and used for the next times (see Table 1). This idea leads to the computational complexity reduction, as discussed above. The proposed method requires a memory block to save the contents of Table 1 . The required memory can be a low capacity memory, because it needs to save only a few numbers, however, this requirement can be mentioned as the drawback of the proposed method.

\section{Simulation results}

In this section, the proposed scheme is compared to the CSLM in terms of PAPR reduction, BER performance, and PSD. The simulation results are based on the IEEE 802.16e standard, with $N=256$, and over-sampling $L=4$. Table 6 shows the simulation parameters used in the analysis.

The CCDF of 4 QPSK-modulated OFDM signals is used to demonstrate the PAPR reduction.

The $\mathrm{CCDF}$ is defined as:
Table 6 Simulation parameters based on IEEE 802.16

\begin{tabular}{ll}
\hline Modulation & QPSK \\
Carrier frequency & $2 \mathrm{GHZ}$ \\
Over sampling & 4 \\
OFDM symbols no. & $10^{6}$ \\
Used subcarrier numbers & 256 \\
Used window for PSD & Hann with 512 Seg. elements
\end{tabular}

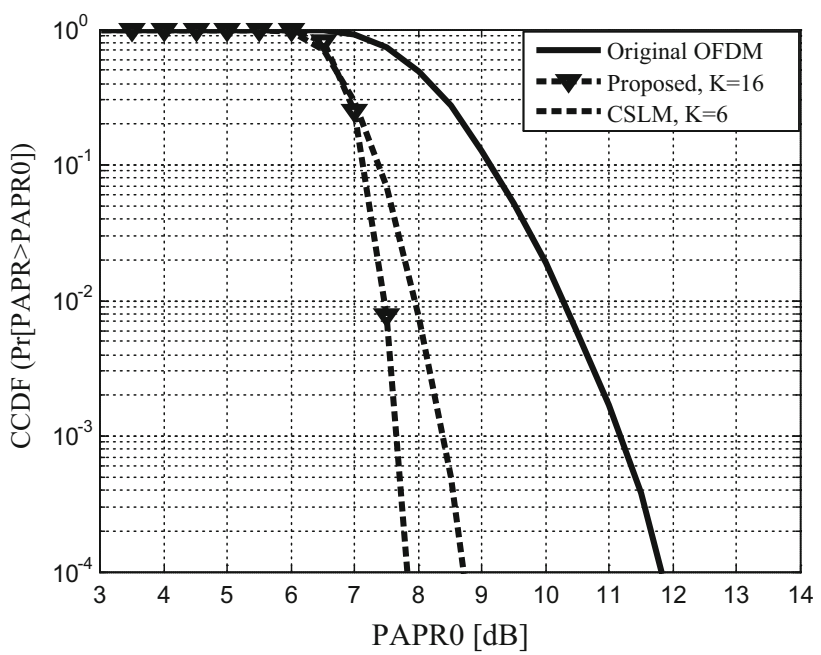

Fig. 7 PAPR comparison of the proposed scheme and CSLM by the same computational complexity (different $K$ values)

$C C D F=P_{r}\left\{P A P R>P A P R_{0}\right\}$

where $P A P R_{0}$ is the set threshold. Figure 6 shows a comparison of PAPR reduction for CSLM, LR-SLM, and Li's method [5] with $K=4,8$, and 16. Because the PAPR is the same for the Kim's method [7] and CSLM, the Kim's method PAPR is not shown in Fig. 6. This figure shows that the proposed method is superior in terms of PAPR reduction, to the Li's method and is almost the same as CSLM and Kim's method, when using one IFFT block (i.e., $V=1$ ).

Figure 7 illustrates the CCDF based on the computational complexity comparison. According to Table 4, the complexity of CSLM with $K=6$ is approximately the same as the proposed method with $K=16$. This figure proves that at the same computational complexity, the proposed method 


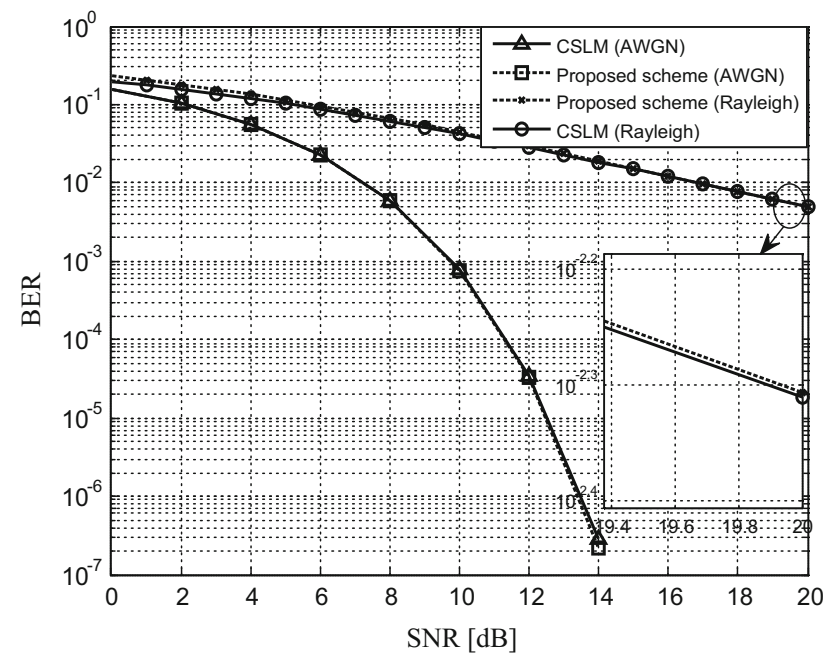

Fig. 8 BER performances of the proposed scheme and CSLM in AWGN and Rayleigh fading channels

has access to more different phase sequences and obtains a higher PAPR reduction performance. In addition to the PAPR reduction comparison, the BER performance in the additive white Gaussian noise (AWGN) and Rayleigh fading channels is examined. The BER as a function of the $E b / N O$ is given by [11]:

$B E R=0.5\left(\sqrt{\frac{E_{b}}{N_{0}}}\right)$

where $E_{b}$ is the energy per modulation bit and $N_{0}$ is the noise spectral density. Figure 8 shows that the proposed method does not impact the BER performance compared to CSLM.

To simulate the scenario in Fig. 1, the ZVE-8G power amplifier, which is suitable for OFDM applications, is applied [9].

Additionally, the DPD method is applied to obviate the distortions generated by the nonlinear amplifier [10]. Figure 9 shows the $\varphi(f)$ which is the PSD for the PA with memory effects. Figure 9a is the PSD of the signal with CSLM and the LR-SLM, without applying the DPD method, and Fig. 9b shows the PSD of the CSLM and LR-SLM with DPD. Because the PAPR is almost the same for the LR-SLM and CSLM, there is no difference in the PSD. Finally, the input OFDM signal is shown in Fig. 9c. Since, this method is a mathematics-based approach, the communication parameters such as PAPR, BER, and PSD are not changed compared to the CSLM method.

\section{Conclusion}

In this paper, a new low complexity scheme using a radixII inverse fast Fourier transform structure is proposed. The

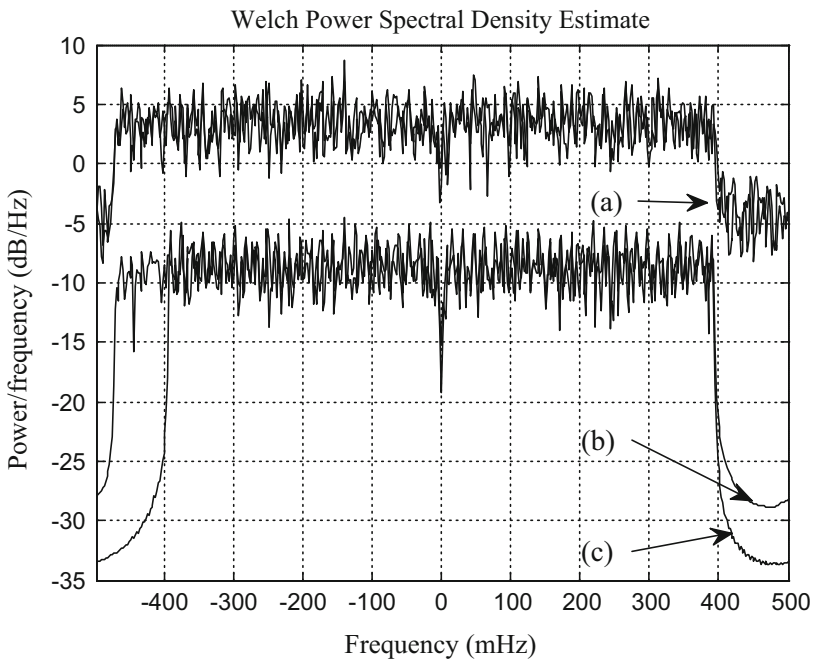

Fig. 9 PSD of the ZVE-8G power amplifier by applying the CSLM and LR-SLM methods. $a$ Without DPD, $b$ with DPD, $c$ input OFDM signal

proposed scheme called LR-SLM is based on $N / 2$ or $N / 4$ different phase multiplications to the input OFDM signal against $N$ random phase sequences of the CSLM scheme. The results show that at approximately the same PAPR reduction, the proposed method provides at least $46.8 \%$ complexity reduction compared to the CSLM with the similar BER and PSD performance. Moreover, LR-SLM outperforms the CSLM by approximately $1 \mathrm{~dB}$ in terms of PAPR reduction at nearly the same complexity. However, a true benefit of this method is in less computational complexity. This scheme is ideal for current and future wireless communication systems such as WIMAX and long-term evolution (LTE) and the mobile consumer electronics which need low power consumption, high speed, and real time communication.

Acknowledgements This work was supported by Universiti Putra Malaysia under the Prototype Development Research Grant Scheme (PRGS) No. 5528700.

\section{Appendix}

In this appendix, the proposed scheme and some equations are described for an $N$-point radix-2 IFFT where $N=8$ and $K=4$. As mentioned above, $K$ is a variable that is specified by the user and determines the computational complexity and PAPR reduction value. If $m^{\prime}=0,1$, then:

$$
\begin{aligned}
& m_{1}=4\left(N / 4-m^{\prime}\right)-1=\{7,3\} \\
& m_{2}=4\left(N / 4-m^{\prime}\right)-2=\{6,2\} \\
& m_{3}=4\left(N / 4-m^{\prime}\right)-3=\{5,1\} \\
& m_{4}=4\left(N / 4-m^{\prime}\right)-4=\{4,0\}
\end{aligned}
$$


Therefore, the input signal is partitioned into the 4 groups by the proposed approach. The required matrices are defined as

$$
\begin{aligned}
& B^{\prime}=\left[\begin{array}{cccccccc}
1 & 1 & 1 & 1 & 1 & 1 & 1 & 1 \\
-1 & -1 & 1 & 1 & 1 & 1 & 1 & 1 \\
j & j & 1 & 1 & 1 & 1 & 1 & 1 \\
-j & -j & 1 & 1 & 1 & 1 & 1 & 1
\end{array}\right]_{4 \times 8}=b_{1} \\
& X^{\prime}=\left[\begin{array}{llllllll}
X_{3} & X_{7} & X_{6} & X_{2} & X_{5} & X_{1} & X_{4} & X_{0} \\
X_{3} & X_{7} & X_{6} & X_{2} & X_{5} & X_{1} & X_{4} & X_{0} \\
X_{3} & X_{7} & X_{6} & X_{2} & X_{5} & X_{1} & X_{4} & X_{0} \\
X_{3} & X_{7} & X_{6} & X_{2} & X_{5} & X_{1} & X_{4} & X_{0}
\end{array}\right]_{4 \times 8} \\
& Y_{1}=\left[\begin{array}{cccccccc}
X_{3} & X_{7} & X_{6} & X_{2} & X_{5} & X_{1} & X_{4} & X_{0} \\
-X_{3} & -X_{7} & X_{6} & X_{2} & X_{5} & X_{1} & X_{4} & X_{0} \\
j X_{3} & j X_{7} & X_{6} & X_{2} & X_{5} & X_{1} & X_{4} & X_{0} \\
-j X_{3} & -j X_{7} & X_{6} & X_{2} & X_{5} & X_{1} & X_{4} & X_{0}
\end{array}\right]_{4 \times 8}
\end{aligned}
$$

The matrix $Y_{1}$ shows that by changing the phase sequences 4 times, only 2 input samples are changed, and the other samples are fixed. Hence, $R_{l}, R_{l+N / 8}, G_{d}$, and $H_{d}$ have to be calculated once, saved, and used for the subsequent rows, whereas in the CSLM method, all similar calculations should be performed during each searching procedure. According to Table 1 , for $q=3$ and $l=0$, the saved parameters are $G_{0}, G_{1}, G_{2}, G_{3}, R_{0}$, and $R_{1}$ where $R_{0}=\alpha_{0}+W^{0} \beta_{0}$ and $R_{1}=\alpha_{0}-W^{0} \beta_{0}\left(\alpha_{0}=X_{5}, \beta_{0}=X_{1}\right) . T_{K, \operatorname{Pr} o-M u l}$ has 2 parts. The first part is related to the calculations of the first row, and the second part is for the other rows of the selected matrix. $T_{K, \operatorname{Pr} O-M u l}$ and $T_{K, C S L M-M u l}$ with $K=4$ and $N=8$, are evaluated as

$$
\begin{aligned}
& T_{K, \operatorname{Pr} o-M u l}=4 \log _{2} 8+(3) \underbrace{\left(\log _{2} 8+4\right)}_{7} \\
& T_{K, C S L M-M u l}=4\left(4 \log _{2} 8\right)
\end{aligned}
$$

Referring to (40) the second part is shown by bold circles in Fig. 4 (i.e., 7 multipliers for each row, except the first row). The following matrix is generated, whit $N=8$ and $K=8$ :

$$
B^{\prime}=\left[\begin{array}{l}
b_{1} \\
b_{3}
\end{array}\right]=\left[\begin{array}{cccccccc}
1 & 1 & 1 & 1 & 1 & 1 & 1 & 1 \\
-1 & -1 & 1 & 1 & 1 & 1 & 1 & 1 \\
j & j & 1 & 1 & 1 & 1 & 1 & 1 \\
-j & -j & 1 & 1 & 1 & 1 & 1 & 1 \\
1 & 1 & 1 & 1 & 1 & 1 & 1 & 1 \\
1 & 1 & 1 & 1 & -1 & -1 & 1 & 1 \\
1 & 1 & 1 & 1 & j & j & 1 & 1 \\
1 & 1 & 1 & 1 & -j & -j & 1 & 1
\end{array}\right]_{8 \times 8}
$$

\section{References}

1. Rodrigues, M. R. D., \& Wassell, I. J. (2006). IMD reduction with SLM and PTS to improve the error-probability performance of nonlinearly distorted OFDM signals. IEEE Transactions on Vehicular Technology, 55(2), 537-548.

2. Baxley, R. J., \& Zhou, G. T. (2007). Comparing selected mapping and partial transmit sequence for PAR reduction. IEEE Transaction on Broadcasting, 53(4), 797-803.

3. Varahram, P., \& Ali, B. M. (2011). Partial transmit sequence scheme with new phase sequence for PAPR reduction in OFDM systems. IEEE Transactions on Consumer Electronics, 57(2), 366371.

4. Ahmed, S., \& Kawai, M. (2013). Interleaving effects on BER fairness and PAPR in OFDMA system. Telecommunication Systems,. doi:10.1007/s11235-011-9557-6.

5. Li, C. P., Wang, S. H., \& Wang, C. L. (2010). Novel low-complexity SLM schemes for PAPR reduction in OFDM systems. IEEE Transactions on Signal Processing, 58(5), 2916-2921.

6. Varahram, P., \& Ali, B. M. (2014). A crest factor reduction scheme based on recursive optimum frequency domain matrix. IEEE Transactions on Consumer Electronics, 60(2), 179-183.

7. Kim, K. H., Jeon, H. B., No, J. S., \& Shin, D. J. (2013). Low complexity selected mapping scheme using cyclic-shifted inverse fast Fourier transform for peak-to-average power ratio reduction in orthogonal frequency division multiplexing systems. IET Соттиnications, 7(8), 774-782.

8. Kazemian, M., Varahram, P., Ali, B. M., \& Mohammady, S. (2015). A low complexity PAPR reduction scheme based on Radix-II IFFT. IEEE international conference on consumer electronics, pp. 420 421.

9. Varahram, P., \& Ali, B. M. (2011). A low complexity partial transmit sequence for peak to average power ratio reduction in OFDM systems. Radio Engineering, 20(3), 677-682.

10. Varahram, P., \& Ali, B. M. (2013). Low complexity partial transmit sequence with complex Gain memory predistortion in OFDM systems. Wireless Personal Communications, 68(3), 1435-1448.

11. Kazemian, M., Varahram, P., Hashim, S. J., Ali, B. M., Mohammady, S., \& Sulaiman, N. (2014). Peak-to-average power ratio reduction based on cross-correlation in OFDM systems. International conference on advanced communications technology, pp. 244-248.

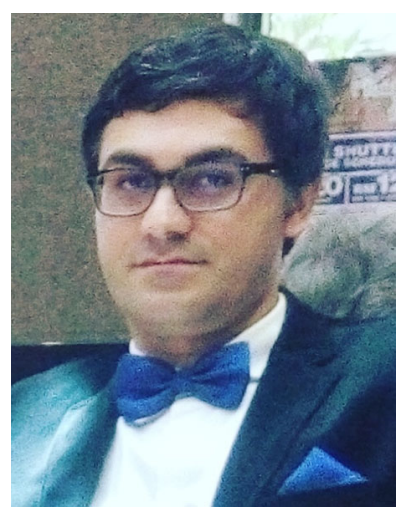

Mohsen Kazemian received his B.Sc. Electrical and Electronics Engineering in 2007, and his M.Sc. in Telecommunications Engineering from Islamic Azad University in 2009. His Ph.D. degree is received from Universiti Putra Malaysia (UPM), on Wireless Telecommunication field at October 2016. His research interest is PAPR reduction in OFDM wireless systems and the Linearization of power amplifiers. He is inventor on one patent in this research area and is a member of IEEE since 2014. 


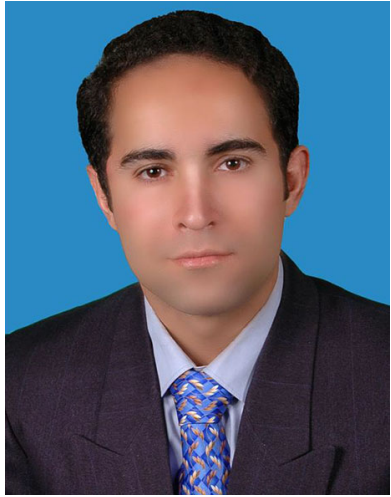

Pooria Varahram received his B.Sc. from Khaje Nasir University of Technology in 2002, his M.Sc. and Ph.D. from Tarbiat Modares University and Universiti Putra Malaysia in 2004 and 2010, respectively. He has more than 5 years of experience in designing and developing a range of electronic and telecommunication related projects. $\mathrm{He}$ was senior lecturer in UPM for 3 years and now is senior postdoctoral researcher with Callan institute, National University of Ireland since August 2015. His research interests are PAPR reduction in OFDM systems, linearization of power amplifiers, microwave power amplifier design.

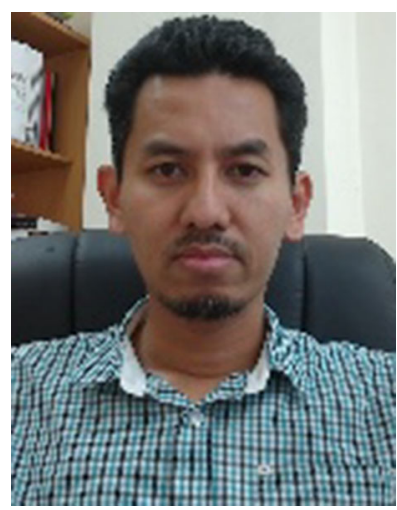

Shaiful Jahari bin Hashim is currently a senior lecturer at the Department of Computer and Communication Systems Engineering, Faculty of Engineering, Universiti Putra Malaysia. He received his Ph.D from Cardiff University, UK (2011), M.Sc from Universiti Kebangsaan Malaysia (2003) and hi B.Eng from University of Birmingham, UK (1998) in the field of electrical and electronics Engineering. His research interest is network security, cloud computing and wireless measurement system.

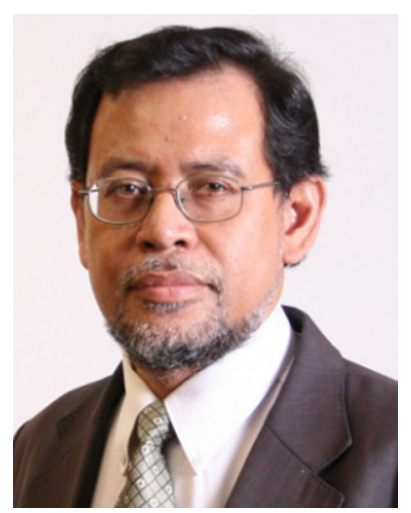

Borhanuddin Mohd Ali obtained his B.Sc. (Hons) Electrical and Electronics Engineering from Loughborough University in 1979; M.Sc. and Ph.D. from University of Wales, Cardiff, UK, in 1981 and 1985, respectively. He became a lecturer at UPM in 1985, and Professor in 2002 and served in various positions in UPM and various external organizations. He is a Senior Member of IEEE and a member of IET and a Chartered Engineer. He served at various positions at ComSoc and Malaysia Section, and IEEE Region 10, and is presently Executive Co-Chair of the ICC2016 Kuala Lumpur. His research interest includes Wireless Sensor Networks, Wireless Resource Management, Mobility, MIMO and OFDM, in which he has published over 100 papers in refereed journals and over 200 conference papers.

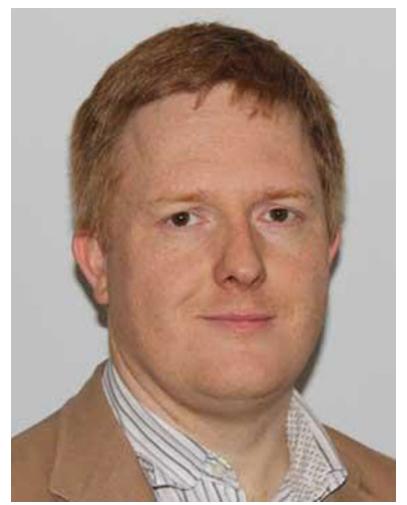

Ronan Farrell received his BE and $\mathrm{Ph} . \mathrm{D}$ from University College Dublin in 1993 and 1998 respectively. In 2008 he became a strand co-leader for sensors networks in an SFI Cluster on advanced Geotechnologies with a focus on wide area wireless sensor networks. Ronan has published over a hundred peerreviewed papers. He holds three patents and licensed technology that has led to the spin-out three companies, Ronan's personal research interests include wireless system design, electronics and radio systems. 